\title{
Criterios de la traducción científica \\ Criterion of Scientific Translation
}

Instructor Mohamad Dakhil Diab: dakhil016@gmail.com

University of Baghdad, Collage of Languages, Department of Spanish Language

( Received on 30/12/2019 - Accepted on 3/2/2020 - Published on 1/6/2020 )

\section{Abstract}

The study stresses number of criterion that can enhance the process of a successful into Spanish scientific translation. The translation of various texts of engineering, medicine, pharmacology, physics, chemistry, mathematics and other sciences which are directly connected with Man's life has been extensively influenced in many of its terms and words by English and relatively a lot of other languages. The study, also, suggests number of the successful methods used to overcome difficulties faced in rendering the text into the target language in a natural and easily understood manner, methods that are considered the cornerstone of a successful scientific translation.

Keywords: Translation, scientific, texts, difficulties, criterion 


\title{
Criterios de la traducción científica
}

\author{
Inst. Mohamad Dakhil Diab \\ Facultad de Idiomas - Depto. de español
}

\section{Resumen}

El presente trabajo de investigación se centra en echar luz sobre los criterios más importantes por los cuales el traductor puede conseguir el total éxito en la traducción de los textos científicos cuyo lenguaje escrito en español se halla influenciado a gran nivel con expresiones y palabras adoptadas de la lengua inglesa y de otras lenguas mundiales, y exponer las dificultades más destacadas que enfrentan a los traductores a la hora de traducir del idioma español al idioma árabe, dando las soluciones más adecuadas a la cabeza de las cueles llegar a un nivel aceptable de la comprensión.

Palabras claves: traducción, textos, científicos, dificultades, criterios.

\section{.Introducción}

Existen varios tipos de traducción, entre los cuales, la traducción de los textos científicos que se refieren a traducir textos farmacéuticos, tecnológicos, médicos y todos los tipos relacionados al respecto que exigen una precisión absoluta de alta calidad y habilidades profesionales que sobre la cual, me refiero a la traducción científica, gira el presente trabajo de investigación, hablando sobre el concepto general del lenguaje científico, las 
dificultades que enfrentan al traductor y los criterio más importantes que llevan a tener una traducción exitosa e ideal.

El objetivo principal del este trabajo de investigación es exponer y aclarar, de una forma sencilla, cómo se traducen los textos científicos cuyo terreno es amplísimo que comprende la ingeniería, la medicina, la física, la biología, la química, la psicología, los productos farmacéuticos y la ciencia veterinaria, que se caracterizan por los complejos argumentos, la específica terminología y temas muy delicados, cuyo traductor debe ser caracterizado por la competencia, las sólidas habilidades, la experiencia y la amplia pasión por el mundo de las ciencias, innovaciones y descubrimiento tecnológicas con el objetivo de llegar a una traducción científica aceptable y comprensible. El texto científico se basa en una objetividad por la cual se elimina cualquier equivocación o ambigüedad, eso quiere decir que el léxico científico, que establece por parámetros asentados en el rigor y la precisión, proporciona una correcta interpretación, lo cual ha sido un motivo de facilitar la tarea del traductor que debe comprender bien el texto antes de emprender traduciendo.

\section{Concepto del lenguaje científico}

El lenguaje científico se trata de ser una lengua de especialidad con propias e independientes características en cuanto a su vocabulario, con menos uso de sintaxis y de morfología. Es un lenguaje mundial tiene como objetivo sacar ventajas, tratando temas relacionados a las ciencias naturales y sus aplicaciones en los ámbitos de la industria, medicina, ingeniería, agricultura, minería, economía, gestión de obras, análisis, piscología, clasificación de razas, ley, turismo, arqueología, historia y geografía (Byrne, 2006: 65). 
El estilo del lenguaje científico se caracteriza por la objetividad, alejarse de la imaginación, de la agitación de las emociones, se recurre más a garantizar la claridad, a la precisión y a ser realista. Utiliza términos técnicos especializados, abreviaturas y símbolos. El objetivo del artículo científico es sacar beneficios por medio de la presentación lógica de las ideas, chocar la mente e interpretar los hechos, y descubrir los misteriosos sin exagerar, y alejarse del vocabulario ornamentado. El lenguaje científico no usa el eufemismo, que se trata de usar un vocablo menos ofensivo que sustituye a otra palabra de mal gusto que puede insultar o referirse a algo no agradable o insultante al oyente, para reducir el impacto del habla, sino usa la palabra tal y como es para indicar algo o hecho tal y como es, como por ejemplo, no usa la el verbo sacrificar en lugar del verbo matar, y no se usa la expresión se fue lejos en vez de morir (Byrne, 2012: 84).

La retórica científica no se eleva a la escala de la retórica literaria. Tiene un significado más simple, definido como un método utilizado por el autor científico para organizar la información en un solo párrafo o en un solo texto de la mejor manera. El lenguaje científico en los textos científicos utiliza varias herramientas, que son un tipo de las características retórica, para lograr precisión y claridad, como márgenes, comentarios, referencias, listas, esquemas, gráficos, imágenes y mapas, que a su vez son propiedades retóricas (Byrne, 2012: 87).

A veces, encontramos que el lenguaje científico usa metáforas cuando sea necesario para ser de un tipo familiar con el objetivo de fortalecer el estilo y hacer el tema vital para hacer interesar al lector, pero no usa los recursos estilísticos, no se recure a la complicación de la sintaxis y el léxico, ni usa los elementos cómicos y trágicos (Gamero Pérez, 1999: 139). 


\section{Estructura lingüística del texto científico}

A. Vocabulario: aunque el lenguaje científico tiene unas fijas palabras y no se cambia con el paso del tiempo, no como sucede con el vocabulario del lenguaje general usado en la vida pública que se cambia según la situación del hablante, lo que ha sido un motivo de hacer el lenguaje literario caracterizar por la claridad y la facilidad de escritura. El vocabulario del lenguaje científico se divide en tres categorías:

1. Vocabulario prestado: son palabras que los científicos han tomado prestadas del lenguaje hablado, dándolas una apariencia científica con el fin de utilizarlas de una nueva manera científica. Estas palabras que, a veces, tienen un significado específico o no está bien claro respecto a su ámbito original o el ámbito nuevo, como por ejemplo, la palabra vida que significa tiempo entre el nacimiento y la muerte de un ser orgánico, que equivale a la palabra (biología) que significa la ciencia que estudia la estructura, la función y el desarrollo de los seres vivos, que se refiere a la ciencia de la vida. Hay otras palabras cuyo significado original tiene un significado específico o más específico, como por ejemplo, la palabra sal que se usa por la gente en varias situaciones a referirse a varios significados, por ejemplo, «En aquel tiempo, dijo Jesús a sus discípulos: Ustedes son la sal de la tierra». Mientras que si se usa en el aspecto científico, especialmente en el campo de la química encontramos que la palabra sal significa compuesto formado por cationes, iones cargados positivamente, enlazados a aniones, iones cargados negativamente, producto típico de una reacción química entre una base y un ácido. Por otro lado, hay palabras que no tienen una relación respecto a su significado en el lenguaje científico y en el lenguaje general, por ejemplo, la palabra fuerza que significa en el uso corriente capacidad para mover o deformar o empujar algo o alguien, con peso o que haga resistencia, 
mientras se usa en el campo de la física en el significado de causa que puede acelerar un cuerpo con masa o deformarlo que en el sistema MKS su unidad es el Newton (Gamero Pérez, 2001: 95).

2. Vocabulario importado: son palabras que han sido traídas de otras lenguas tanto las antiguas como las modernas al lenguaje científico, usadas sin ningún cambio en cuanto a su pronunciación o su significado, por ejemplo, existen palabras que pertenecen al antiguo idioma griego, como átomo que significa en el campo de la física y química partícula más pequeña posible de un elemento químico que conserva su identidad o sus propiedades. Otras son pertenecientes al árabe como la palabra álgebra que significa en el campo de la matemática rama de las matemáticas relativa a estructura, relaciones y cantidades. En el álgebra se utilizan tanto símbolos a los números como a sus operaciones y relaciones. Otras pertenecen al idioma francés, como la palabra nicotina que significa en campo de la química alcaloide encontrado comúnmente en la planta del tabaco, de color acre que en pequeñas dosis es un estimulante adictivo, en grandes dosis es toxico y es utilizado a menudo en insecticidas). Otras pertenecen al idioma italiano, como la palabra malaria que significa en el campo sanitario enfermedad producida por parásito vector de la malaria, que los síntomas son muy variados, empezando con fiebre 8 a 30 días posteriores a la infección y acompañada, o no, de dolor de cabeza, musculares, diarrea, decaimiento y tos (García Yebra, 2004: 25-36).

3. Vocabulario inventado: son palabras trazadas por los científicos durante su investigación para describir sus descubrimientos y sus teorías científicas. Son palabras numerosas y van en línea creciente por causa de la falta de la capacidad de una determinada lengua respecto a contenerlas. Estas palabras pueden haber sido bien conocidas y bien definidas por su autor al comienzo 
de su aparición, lo cual ha sido un motivo de ser palabras eternas respecto al uso científico, como la palabra vitamina que significa en el ámbito de la biología y la química compuesto orgánico heterogéneo que se encuentra en los animales en pequeñisimas cantidades, pero que son necesarios para el correcto funcionamiento orgánico. Actúan como coenzimas y grupos prostéticos de las enzimas, recibiendo sus nombres distintivos mediante letras mayúsculas (Gonzalo García, 2004: 54).

B. Gramática: las fórmulas gramaticales más utilizadas por el lenguaje científico para conseguir expresar el material científico de una forma tan clara y tan comprensible, son:

1. Voz pasivo: esta fórmula se usa, con frecuencia, porque el lenguaje científico se interesó por el efecto y el evento sin importar la causa en muchos casos.

\section{Ejemplo:}

- El corazón es constituido por cuatro cavidades.

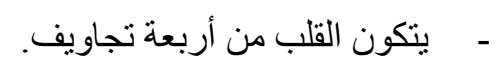

2. Tiempos verbales del modo indicativo: que se usan con el fin de garantizar el carácter universal, sobre todo, se usa el presente que es la forma más común en el lenguaje científico, en la tercera persona, porque expresa el estado científico actual del conocimiento científico, y se corresponde a la naturaleza del tema científico respecto a explicar los fenómenos, los hechos y las pruebas científicas. 


\section{Ejemplo:}

- Por muchos científicos actuales se admite que el Universo se halla en continua expansión.

$$
\text { - يرى الكثير من العلماء في الوقت الحالي ان الكون في حالة نوسع مستمر. }
$$

3. Verbos copulativos: que se usan, a veces, con el fin de dar definiciones concretas, aunque a principios del siglo anterior se consideraban adecuadas las estructuras oracionales muy complejas de utilizar cada vez más oraciones simples ampliadas, en el que las ampliaciones aparecen sobre todo en el terreno de los atributos y de las determinaciones adverbiales (Hurtado Albir, 2001: 75).

\section{Ejemplo:}

- El corazón es el órgano principal del sistema circulatorio. Su función es propulsar la sangre a todo el cuerpo.

$$
\text { ـ القلب هو الجهاز الرئيسي في الدورة الدموية. وتتمثل مهمته في دفع الدم في جميع أنحاء الجسم. }
$$

4. Gerundio: que se usa con fin de dar un estado de continuidad.

\section{Ejemplo:}

- La noción de un Universo en expansión, es decir que las galaxias que lo forman se van alejando o separando cada vez más.

- تصور حالة تمدد الكون, يعني أن المجرات التي يتكون منها تتحرك بعيدا أو تتفصل عنه باستمرار. 
5. La importancia de los signos de puntuación respecto a escribir los textos científicos, que ayudan al lector a comprender el material científico escrito sin ambigüedad o confusión, ya que a nivel de la oración, necesitamos una compresión sintáctica, en el sentido de una concentración de la información, que corresponde a la tendencia científica que sin la existencia de buena ortografía nunca se puede conseguir (Santoyo Mediavilla, 1983: 53).

\section{Ejemplo:}

- La rápida expansión de esta bola gaseosa incandescente se acompañó de una serie de procesos complejos que provocaron la agrupación de las partículas elementales en átomos. A medida que proseguía la expansión, la temperatura fue descendiendo y los átomos de los elementos de un elevado punto de fusión, tales como hierro y silicio ${ }_{2}$ cristalizaron en forma de polvo cósmico que quedó flotando en el seno de una masa gaseosa formada principalmente por hidrógeno y helio.

$$
\begin{aligned}
& \text { - ير افق التوسع السريع في هذه الكرة الغازية المتوهجة سلسلة من العمليات المعقدة التي تسبيت في }
\end{aligned}
$$

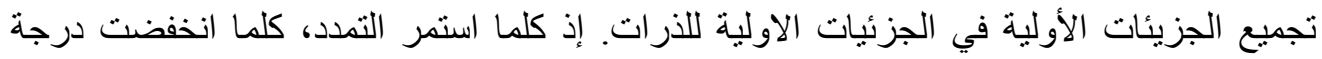

$$
\begin{aligned}
& \text { الحرارة وتبلورت ذرات عناصر ذات درجة انصهار عالية، كالحديد والسليكون في شكل غبار كوني، } \\
& \text { تطفو داخل كتلة غازية تتثكل أساسًا بواسطة الهيدروجين و الهيليوم. }
\end{aligned}
$$

Por lo tanto, vemos que los signos de puntuación organizan los elementos del discurso, sin los cuales resulta, normalmente, la ambigüedad, por ejemplo una coma puede cambiar el sentido de un cierto texto, como podemos observar en los siguientes ejemplos:

- No, espere $\rightarrow$ (una pausa)

-

- No espere $\rightarrow$ (negativo) 
- $\quad$ No queremos saber $\rightarrow$ (negativo) -

- No, queremos saber $\rightarrow$ (cambiar de opinión) -

\section{Dificultades de la traducción científica}

\section{Elipsis y uso de pronombres}

\section{Ejemplo:}

- La turbina es una rueda hidráulica provista de paletas como un molino de viento sobre las cuales (paletas) el agua ejerce su energía por presión, haciéndola (la turbina) girar. Es (la turbina) una máquina poco complicada que también puede (la turbina) ser movida por gas o pavor. Se usa (la turbina) mucho en la industria, pues (la turbina) genera una gran energía al aprovechar mиy bien la fuerza que hace mover las paletas. Las grandes turbinas pueden producir centenares de miles de caballos de vapor.

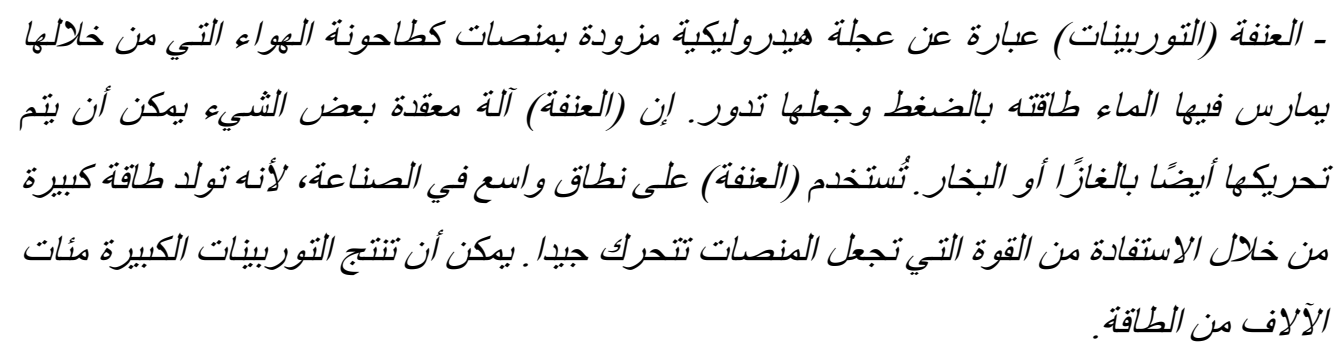

Vemos en el ejemplo anterior, el de la turbina, que es un texto de contenido científico procedente de la física, dirigido a los especialistas o profesionales de este campo, que es un texto extraído de la enciclopedia de la palabra a la imagen, conocer, ver y aprender, que es un texto lleno del fenómeno lingüístico llamado la elipsis que, gramaticalmente, se trata de 
suprimir, sin contradecir las normas gramaticales, una o puede ser más de una palabra que supone que no ocasiona ambigüedad en la oración con el fin de construir las oraciones con exactitud, con coherencia y sin pleonasmo. Respecto a los tipos de la elipsis, existen dos: la verbal que se trata de eliminar el verbo. Mientras el segundo es nominal que se trata de omitir el pronombre o el nombre (Lang, 1997: 83).

Por lo tanto, el traductor, que tiene que contar con las herramientas genéricas de consulta más habituales como los diccionarios, las gramáticas, los manuales de estilo, las fuentes de documentación e información general, debe entender bien el texto, dándose cuenta de qué se trata la elipsis y luego empieza a traducir para llegar a una traducción aceptable careciente de las ambigüedades. A veces no es suficiente una primera lectura para entender el texto, por eso, hay que navegar en los elementos del texto científico, a partir de la redacción, los anacolutos, el ritmo, el léxico, la terminología especializada, los objetivos, la justificación a las dudas y las preguntas, porque con el fin de resolver con éxito los problemas de traducción de los textos científicos es importante conocer las diversas herramientas de que se puede disponer y estar bien informado respecto a la difusión de los avances científicos, así como el intercambio de información, experiencias y conocimientos de los expertos a escala mundial (Galán Rodríguez, 2002: 92).

\section{Uso de sinónimos}

Aunque, teóricamente, que el léxico científico adquiere unas propias propiedades semánticas y neutrales que lo hacen diferente en comparación con el léxico común que es caracterizado por la polisemia, la sinonimia y la ambiguiedad, que estos fenómenos son menos habitual en el léxico científico, pero eso no significa que el léxico científico carece de los casos de sinonimia, 
de polisemia, de dependencia contextual y de connotación, como por ejemplo lo que aparece en los casos del uso de las abreviaturas que se convierte, a veces, en una fuente de sinonimia (Rodríguez Adrados, 1975: 54).

Las abreviaturas es un mecanismo que funciona tanto en el léxico común como en el científico que es un medio escrito cuyo objetivo es reducir un sintagma o una palabra a una o varias de sus letras competentes, como por ejemplo, $(g . \rightarrow$ gramo $),($ c.p.s $\rightarrow$ ciclos por segundo $),(\mathrm{cm} . \rightarrow$ centímetro $)$ (Martín Camacho 2007: 241-245).

Por ejemplo, encontramos que la abreviatura científica del (gramo $\rightarrow g$ ) según la normativa del Sistema Internacional de Unidades, mientras coloquialmente la gente emplea la abreviatura del gramo en la forma singular ( $g r$.) o, a veces, en el plural (grs.), por lo tanto, es muy frecuente que encontramos en los centros comerciales que hay carteles que anuncian el precio de un cierto producto de $300 \mathrm{gr}$., pero encontramos que el mismo producto será anunciado en otro centro comercial usando la abreviatura científica de $(g$.$) en lugar de (g r$.$) , por lo tanto, el traductor debe tener en$ cuenta que la abreviatura ( $g$.) que está usada a nivel puro científico es el sinónimo de la abreviatura de ( $g r$.) usada a nivel del habla común (Alvar Ezquerra, 1983: 65).

\section{Criterios del éxito de la traducción científica}

\section{Capacidad comunicativa y entender el texto original}

Tradicionalmente, la traducción se ha basado en la equivalencia, pero esta visión ya está un poco cambiada. Lo que es importante en la traducción de un texto científico, hoy en día, no es solo la equivalencia entre palabras, sino la traducción de las ideas principales, en relación con el fondo cultural de 
la lengua meta. Al traducir los textos científicos, primero se debe tener en cuenta la precisión del texto traducido, en términos de cómo transmite el objetivo del autor del texto de origen al lector del texto traducido. Por lo tanto, el texto traducido será capaz de contribuir instrumentalmente al área científica (Martin Camacho, 2007: 34).

La capacidad comunicativa que se refiere a comprender el texto original y de expresarse tanto oralmente como por escrito en el idioma castellano y la lengua árabe, consiste en el primer criterio que es, justamente, lo que hace posible, que el texto científico puede ser trasmitido del español al árabe, por lo tanto, no puede resultar ninguna traducción tan correcta sin entender lo que contiene el texto tanto a nivel explicito como a nivel implícito. Además de dominar el lenguaje especializado, es decir que tenga la capacidad de buscar, usar e integrar la información, capacidad de aplicar los conocimientos a la práctica, conocimiento activo de la lengua española, técnicas de traducción y análisis de textos, conocimiento gramatical necesario para la lectura y comprensión de textos escritos en un idioma moderno, conocimiento del fenómeno lingüístico en todas sus dimensiones, conexiones interdisciplinarias, conocimiento de la estructura profunda de la lengua española (Martin Camacho, 2006: 27).

\section{Llegar a un nivel aceptable respecto a comprender el texto original y re-expresar adecuadamente}

Alcanzar un nivel aceptable de comprensión es algo muy importante para la traducción de los textos científicos, ya que existen unas situaciones en las que observamos justamente que el léxico se caracteriza, por un lado, por ser comprensible, pero, por otro, no responde a las necesidades funcionales del traductor, por eso, a veces resulta que una primera lectura al texto original no es suficiente para entender el texto, por lo tanto, hay que llegar a un nivel 
aceptable de la comprensión que puede cubrir las necesidades y los criterios científicos y lingüísticos del traductor para llegar a una traducción aceptable (Martin, Camacho, 2004: 76).

Por otro lado, el traductor debe expresar igual justamente a lo existe en los textos científicos, eso quiere decir que transferir la misma estructura del mismo sentido pero por vía de usar elementos diferentes de la otra lengua a la que se hace la traducción, teniendo en cuenta que los textos científicos que por su naturaleza no admiten interpretaciones doble, las palabras aparecen en sus significados principales, se excluyen las acepciones traslaticias y metafóricas, el modo indicativo de los verbos no se emplea en sentido modal y el orden de las palabras en su mayoría es directo (Gutiérrez Rodilla, 1998: 46).

\section{Conclusión}

1. El texto científico se refiere a los estudios, los artículos, textos divulgativos y especializados que tratan de la aplicación práctica del conocimiento y del saber científico. Es caracterizado por ser objetivo de la física del entorno como por ejemplo las ciencias naturales exactas, las físicas y todos los otros tipos científicos vinculados al respecto que representan un saber teórico.

2. La estructura del texto es caracterizada por el uso de la exposición y de la explicación de los métodos, las definiciones, los conceptos, las clasificaciones y las instrucciones técnicas que puede ser organizada en forma de secuencia temporal, causa y efectivo, problema y solución, pregunta y repuesta, descriptiva, enumerativa, planeamiento de las teorías, hipótesis y el análisis. 
3. Con respecto a la estructura lingüística, encontramos que a causa de que el texto científico presenta los rasgos de la claridad, la objetividad, la universalidad y la precisión, pues, se construye, con frecuencia, con el modo de indicativo, formas verbales de obligación, formas no personales del verbo, pasivas, impersonales, sustantivos para expresar las cualidades y los fenómenos, los adjetivos especificativos y descriptivos, prestar palabras de otras lenguas, abreviaturas, palabras nuevas inventadas por los mismos descubridores y palabras de uso habitual.

4. Comprender el texto original bien es la piedra angular en la traducción de los textos científicos, por lo tanto, una sola lectura al texto no es suficiente para llegar a un nivel aceptable de comprender el texto original, ya que, a veces, el traductor siente que ya tiene comprendido todo el texto original, pero a la hora de traducir, siente incapaz de re-expresar adecuadamente.

5. Con el fin de llegar a una traducción ideal respecto al texto científico, el traductor tiene que tener varias cualidades, entre otras la competencia, la experiencia y la actualización continua de la información científica, que se consideran como factores importantísimos a fin de prestar una traducción científica de alta calidad. 


\section{Bibliografía}

1. ALVAR EZQUERRA, M. y MIRÓ DOMÍNGUEZ, A. (1983): Diccionario de siglas y abreviaturas, Madrid: Alhambra.

2. BYRNE, J. (2006). Technical Translation: Usability Strategies for Translating Technical Documentation. Berlín: Springer.

3. BYRNE, J. (2012). Scientific and Technical Translation Explained. A Nuts and Bolts Guide for Beginners. Manchester: St. Jerome.

4. GAMERO PEREZ, S. \& A. Hurtado Albir. (1999). La traducción técnica y científica. En: A. Hurtado Albir (Ed.) Enseñar a traducir. Madrid: Edelsa.

5. GAMERO PEREZ, S. (2001). La traducción de textos técnicos: descripción y análisis de textos (alemán-español). Barcelona: Ariel.

6. GARCIA YEBRA, Valentín. (2004). Sobre la formación de términos técnicos. En: C. Gonzalo García \& V. García Yebra (Eds.) Manual de documentación y terminología para la traducción especializada. Madrid: Arco.

7. GONZALEZ GARCIA, C. \& V. García Yebra (Eds.) (2004). Manual de documentación y terminología para la traducción especializada.

8. Madrid: Arco.

9. HURTADO ALBIR, A. (2001). Traducción y traductología. Introducción a la traductología. Madrid: Cátedra.

10. GALÁN RODRÍGUEZ, C. y MONTERO MELCHOR, J. (2002): El discurso tecnocientífico: la caja de herramientas del lenguaje, Madrid: Arco/Libros.

11. GARCÍA GALLARÍN, C. y GARCÍA GALLARÍN, C. (1997): Deonomástica hispánica: vocabulario científico, humanístico y jergal, Madrid: Complutense. 
12. GUTIÉRREZ RODILLA, B. M. (1998): La ciencia empieza en la palabra. Análisis e historia del lenguaje científico, Barcelona: Península.

13. LANG, M. F. (1997): Formación de palabras en español, Madrid: Cátedra.

14. MARTÍN CAMACHO, J.C. (2004): El vocabulario del discurso tecnocientífico, Madrid: Arco/Libros.

15. MARTÍN CAMACHO, J.C. (2004): "Los procesos neológicos del léxico científico. Esbozo de clasificación”, Anuario de Estudios Filológicos.

16. MARTÍN CAMACHO, J.C. (2007): "La creación de términos científicos mediante procedimientos no morfemáticos", Anuario de Estudios Filológicos.

17. RODRÍGUEZ ADRADOS, F. (1975): "La lengua en la ciencia contemporánea y en la filosofía actual”, Estudios de semántica y sintaxis, Barcelona: Planeta.

18. SANTOYO MEDIALVILLA, J. C. (1983). La cultura traducida. Lección inaugural del curso académico 1983-84. León: Universidad de León. 


\section{معاييز الترجمة العلمية

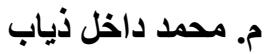

جامعة بغداد ـ كلية اللغات ـ قسم اللغة الاسبانية

خلاصة البحث

يسلط البحث الضوء على أهم المعايير الاساسية التي يستطيع المترجم عن طريقها تحقيق النجاح

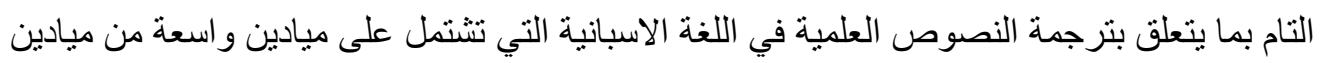
الحياة: كالهندسة والطب و الصيدلة و الفيزياء و الكيمياء و الرياضيات و غير ها من العلوم الاخرى ذات العلاقة الوطيدة بحياة الانسان, و التي على ما يبدو قد تأثرت كلماتها وتعابير ها كثير ا باللغة الانجليزية ولغات العالم الأخرى، فضلا عن تسليط الضوء على أهم الصعوبات التي يواجهها المترجم عند

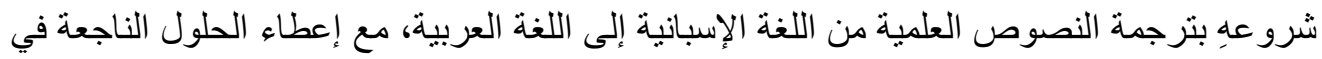

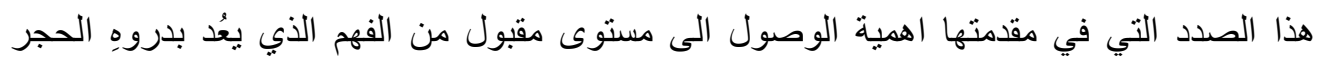
الاساس في نجاح عملية ترجمة النصوص العلمية التي تمتاز بأهميتها الكبيرة في حياة الفرد. الكلمات المفتاحية: الترجمة, النصوص, العلمية, صعوبات برجهات, معايير

\section{Sobre el autor}

Mohamed Dakhi Diab Lillo al-Tamimi. Soy un profesor en el Departamento de Español de la Facultad de Idiomas de la Universidad de Bagdad. He traducido varios libros del español al árabe y al revés, entre los cuales, mencionamos el libro de (El príncipe que buscaba la verdad, Magnificas imágenes de la civilización de Bagdad durante la época abasí, y muchos otros libros).

Email: dakhil016@gmail.com 